\title{
Design and Simulation of EMI Filter Based on LTCC Process Technology
}

\author{
Peng Lei \\ College of Communication Engineering \\ Chengdu University of Information Technology \\ ChengDu, China
}

\author{
Wanting Zhang \\ College of Communication Engineering \\ Chengdu University of Information Technology \\ ChengDu, China
}

\begin{abstract}
This electronic document is a "live" template. The various components of your paper [title, text, heads, etc.] are already defined on the style sheet, as illustrated by the portions given in this document. Do not use special characters, symbols, or math in your title or abstract. The authors must follow the instructions given in the document for the papers to be published. You can use this document as both an instruction set and as a template into which you can type your own text.
\end{abstract}

Keywords: component; formatting; style; styling; insert (Minimum 5 to 8 key words)

\section{INTRODUCTION}

With the development of science and technology, the frequent use of a large number of electronic components, has been resulting in electronic environmental pollution, electromagnetic interference (EMI), which is increasingly serious [1-3]. EMI filters for different frequency bands have been widely used in different application scenarios. On the other hand, the miniaturization and integration of electronic components puts higher requirements on the production process. LTCC process technology is one of the advanced three-dimensional packaging technologies, characterized by excellent high frequency characteristics, excel-lent transmission performance, high reliability and strong stability [4-7]. In this paper, the three-dimensional electromagnetic field simulation software HFSS is used to extract the values of the inductor and capacitor parameters. Based on the LTCC process, the third-order $\pi$-type Butterworth low-pass EMI filter is designed and simulated. Simultaneously, the Sparameters are analyzed, which provides a valuable reference for the development of related practical EMI filters.

\section{DESIGN FOR EMI FILTER}

\subsection{Filter structure}

The mainstream LC passive filter structure mainly includes T-type and $\pi$-type structures. This paper uses a 3rd-order $\pi$ type structure, shown in Figure 1. The Butterworth low-pass filter with a cut-off frequency of $310 \mathrm{MHz}$ is obtained by simulation software Advanced Design system (ADS). Table 1 shows the value of the filter's inductor and capacitor.

\begin{tabular}{|c|c|c|}
\hline Parameter & Value & Unit \\
\hline L1 & 50.68629 & $\mathrm{nH}$ \\
\hline $\mathrm{C} 1$ & 10.13726 & $\mathrm{pF}$ \\
\hline
\end{tabular}

Table1 The value of the inductor and capacitor

\subsection{Design for inductor and capacitor}

The capacitor is in the form of a Vertical-InterdigitalCapacitor (VIC), as shown in Figure 2. The vertical panel size of each layer is $0.8 \mathrm{~mm} \times 0.8 \mathrm{~mm}$. Figure 3 shows the vertical panel parameter values. Equation (1) gives the effective value of the capacitor by the conversion of the scattering parameters [8]:

$$
\mathrm{L}=\frac{-1}{2 \cdot \pi \cdot f \cdot \operatorname{lm}(Y 11)}
$$

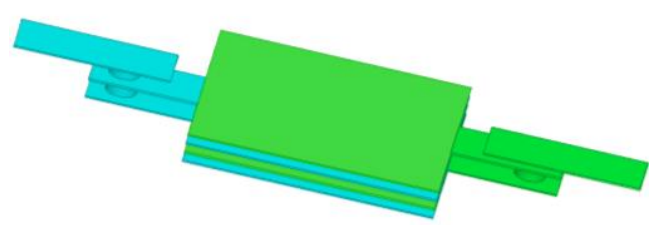

Figure 2 The VIC structure capacitor

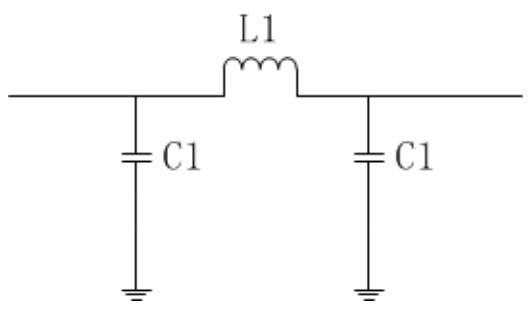

Figure 13 rd-order $\pi$-type structure filter 


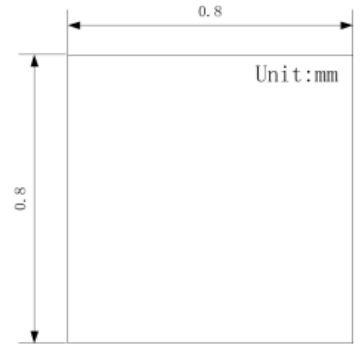

Figure 3 Vertical panel parameters of the capacitor

The inductor uses a spiral U-shaped structure as shown in Figure 4. Its long side is $0.8 \mathrm{~mm}$ and the short side is $0.1 \mathrm{~mm}$. Figure 5 shows its U-shaped structural parameters. Equation (2) gives the effective value of the inductance by the conversion of the scattering parameters [9]:

$$
\mathrm{C}=\frac{-1}{2 \cdot \pi \cdot f \cdot i m(z 11)}
$$

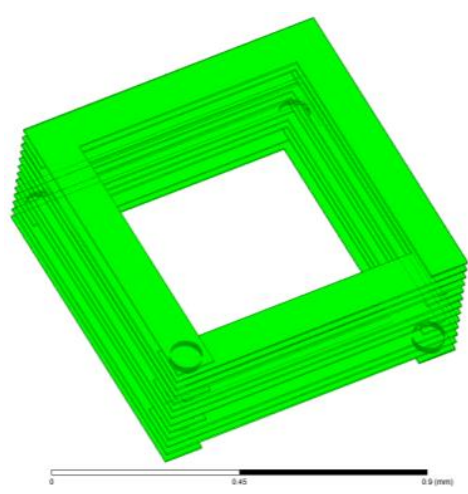

Figure 4 The U-shaped spiral inductor

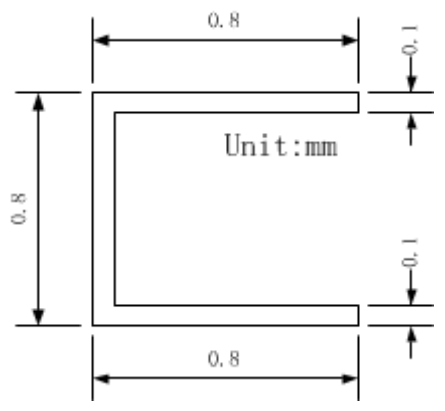

Figure 5 The U-shaped structural parameters

The filter designed in this paper uses the ULF900 ceramic material developed by Ferro and the dielectric constant is 90 . The casting thickness is determined to be $0.024 \mathrm{~mm}$ according to the LTCC process requirements. The conductive paste has a silver thickness of $0.006 \mathrm{~mm}$ and the radius for via hole is $0.04 \mathrm{~mm}$. By continuously adjusting the number of inductor layers and the number of vertical panel layers of the capacitor, the simulation value is close to a predetermined value. Figure
6 gives the varying curve of the effective value of the inductor with frequency. At a frequency of about $310 \mathrm{MHz}$, the equivalent inductor value is about $57.2 \mathrm{nH}$. At a frequency of about $500 \mathrm{MHz}$, the self- resonance happens and the inductor changes from inductive to capacitive. Figure 7 shows the varying curve of the effective value of the capacitor with frequency. At a frequency of about $310 \mathrm{MHz}$, the equivalent capacitor value is about $12.01 \mathrm{pF}$. At a frequency of about $1200 \mathrm{MHz}$, the self- resonance happens and the inductor begins to change from capacitive to inductive.

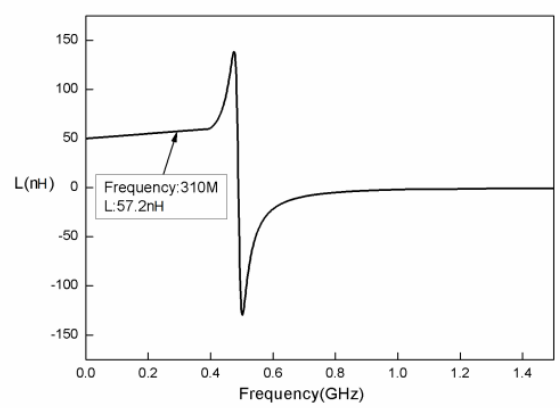

Figure 6 The varying curve of the effective value of the inductor with frequency++

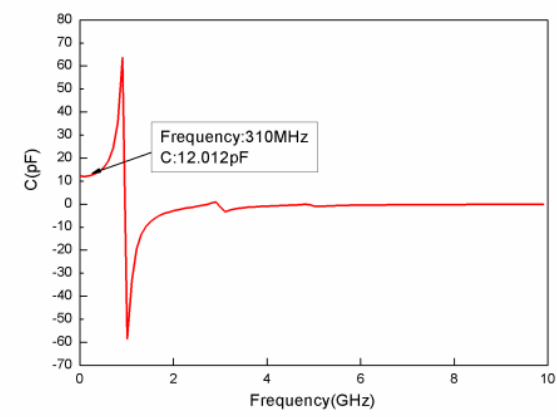

Figure 7 The varying curve of the effective value of the capacitor with frequency

\section{SIMULATION FOR EMI FILTER}

After simulating and analyzing the capacitor and inductor parameters, it is determined that the number of U-shaped spiral inductor layers is 14 , and the number of vertical panel capacitor layers is 4 layers. A 3rd-order $\pi$-type Butterworth low-pass EMI filter is designed by the combination of LC inductors and capacitors. Figure 8 shows the physical model of the EMI filter, which size is $0.9 \mathrm{~mm} \times 0.9 \mathrm{~mm} \times 0.8 \mathrm{~mm}$. Figure 9 shows the varying curve of insertion loss and return loss with frequency for EMI filter. The cut-off frequency is about $340 \mathrm{MHz}$ with the insertion loss of $3.31 \mathrm{~dB}$, and the outof-band rejection is greater than $40 \mathrm{~dB}$ at frequency of about $1.1 \mathrm{GHz}$. The filter cut-off frequency simulation value does not reach the predetermined value of $310 \mathrm{MHz}$, which may be due to the coupling effect between the capacitive and 
inductive components, or may be due to parasitic effects among the inductor, the capacitor and the ground.

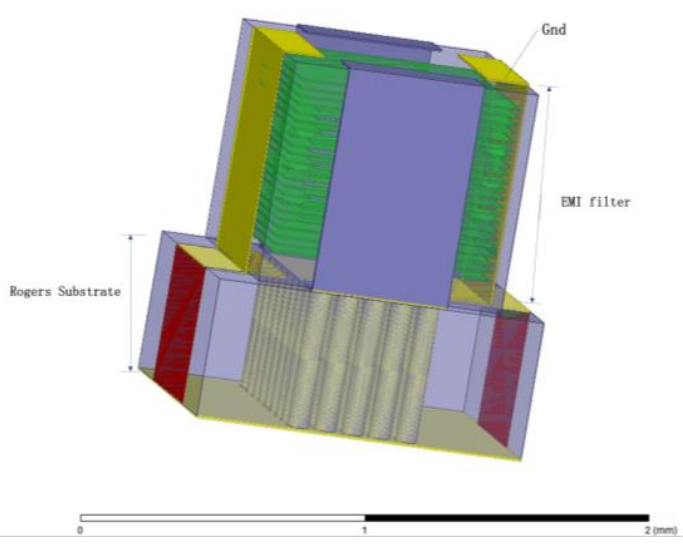

Figure 8 Physical model of the EMI filter

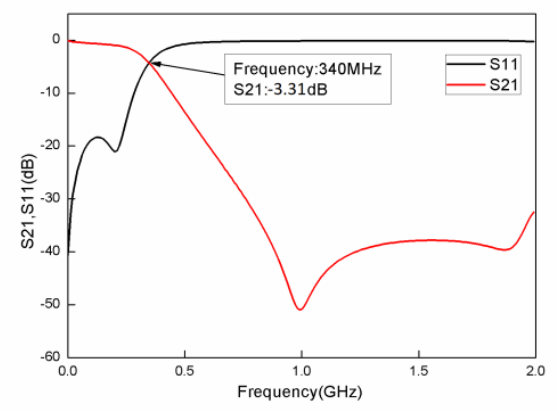

Figure 9 The varying curve of insertion loss and return loss with frequency for EMI filter

\section{CONCLUSION}

Through the 3D electromagnetic field simulation software HFSS, the design and simulation of the 3rd-order $\pi$-type Butterworth low-pass EMI filter based on LTCC technology shows that: 1 there are certain coupling effects and parasitic effects between the multilayer capacitor and the inductor. $2 \mathrm{~A}$ low-pass EMI filter with a cut-off frequency of $340 \mathrm{MHz}$ and an out-of-band rejection of more than $40 \mathrm{~dB}$ at frequency of about $1.1 \mathrm{GHz}$ is successfully designed and simulated, which provides a valuable reference for the development of related devices.

\section{REFERENCES}

[1] Omata, Shinpei, and T. Shimizu . "Design Method for EMI Filters Connected to Both dc and ac ports of a Residential-Use Photovoltaic Power Conditioner." Electrical Engineering in Japan 197.2(2016):56-67.

[2] Jeddi, Javad , and A. A. Katbab . "The electrical conductivity and EMI shielding properties of polyurethane foam/silicone rubber/carbon black/nanographite hybrid composites." Polymer Composites (2017).

[3] Biswas, Sourav, et al. "Rational Design of Multilayer Ultrathin Nano-Architecture by Coupling of Soft Conducting Nanocomposite with Ferrites and Porous Structures for Screening Electromagnetic Radiation." ChemistrySelect 2.3(2017):1094-1101.

[4] Ma, Mingsheng, et al. "A novel wireless gas sensor based on LTCC technology." Sensors \& Actuators B Chemical 239(2017):711-717.

[5] Chin, Kuo Sheng, et al. "LTCC Multilayered SubstrateIntegrated Waveguide Filter With Enhanced Frequency Selectivity for System-in-Package Applications." IEEE Transactions on Components Packaging \& Manufacturing Technology 4.4(2017):664-672.

[6] Jin, Huayan, et al. "60-GHz LTCC Differential-Fed Patch Antenna Array With High Gain by Using SoftSurface Structures." IEEE Transactions on Antennas \& Propagation 65.1(2017):206-216.

[7] Chen, Jian Xin, Y. Zhan, and Q. Xue. "Novel LTCC Distributed-Element Wideband Bandpass Filter Based on the Dual-Mode Stepped-Impedance Resonator." IEEE Transactions on Components Packaging \& Manufacturing Technology 5.3(2017):372-380.

[8] Bo, Zhou, W. Sheng, and Y. Zheng. "Miniaturized Lumped-Element LTCC Filter With Spurious Spikes Suppressed Vertically-Interdigital-Capacitors." IEEE Microwave \& Wireless Components Letters 24.10(2014):692-694.

[9] Ma, Yan Bing , et al. "Design of a LTCC Low Pass Filter." Advanced Materials Research 403-408(2011):17041707. 